\title{
The courtship song of Drosophila santomea and a comparison to its sister species D. yakuba (Diptera: Drosophilidae)
}

\author{
ERIC T. WATSON ${ }^{1}$, EMma RODEWALD ${ }^{2}$ and JERry A. COYNE ${ }^{2}$ \\ ${ }^{1}$ Department of Biological Sciences, Louisiana State University, Baton Rouge, LA 70803, USA \\ ${ }^{2}$ Department of Ecology and Evolution, The University of Chicago, 1101 E. 57 Street, Chicago, IL 60637, USA; \\ e-mail: j-coyne@uchicago.edu
}

Key words. Drosophilidae, behavior, courtship song, interpulse interval, Drosophila, sexual isolation

\begin{abstract}
We describe the male courtship song of the newly-discovered species Drosophila santomea, endemic to the island of Saõ Tomé and the sister species of the widely distributed $D$. yakuba, which also inhabits the island. The song of $D$. santomea resembles that of D. yakuba in lacking a "sine" song, but the interpulse interval (IPI) in the "pulse" song is about $67 \mathrm{~ms}$ for the former species and $110 \mathrm{~ms}$ for the latter. This is the largest difference in IPI observed between any two sister species in the D. melanogaster subgroup, and may play a role in the strong sexual isolation between these two species.
\end{abstract}

\section{INTRODUCTION}

Male courtship in Drosophila is often elaborate (Spieth, 1952; Bastock \& Manning, 1955), involving wing displays and vibrations, with the latter producing audible courtship "songs" (Sturtevant, 1915; Bennet-Clarke \& Ewing, 1967; for a recent review see Tauber \& Eberl, 2003). Most species in the well-studied Drosophila melanogaster subgroup (Fig. 1) have three types of song in their repertoire: "sine song" "pulse song", and "thud song" (Von Schilcher, 1976; Demetriades et al., 1999).

Pulse and thud songs both consist of sharp pulses separated by an interpulse interval (IPI), although these two types of song differ in how they are produced. Pulse songs are generated by the repetitive vibration of a single wing, usually the one closest to the female during courtship, which is extended horizontally to the body of the fly at an angle of $90^{\circ}$ or slightly more. The trailing edge is slightly lowered and vibrated (Bennet-Clarke \& Ewing, 1967), making a noise that, when amplified for the human ear, sounds like a purring cat. In D. melanogaster, D. simulans (Kyriacou \& Hall, 1982), and D. yakuba (Demetriades et al., 1999), the length of the average IPI in pulse songs also changes slightly and predictably between successive pulse bouts, with this change itself forming a long sinusoidal pattern called the "Kyriacou-Hall" cycle (Kyriacou \& Hall, 1982). Evidence for IPI song cycles has also been seen in $D$. persimilis (Noor \& Aquadro, 1998).

The thud song of D. melanogaster-group species sounds like a motorboat when amplified, and is produced by a rapid scissoring action of both wings held at an angle of about $40^{\circ}$ away from the male's abdomen (Demetriades et al., 1999). Thud songs may consist of anywhere from one to several pulsations produced at an irregular frequency. Typically, pulse and thud songs are produced in distinct bursts but, on occasion, a pulse song will begin and then change quickly to a thud song or vice versa (Demetriades et al., 1999).

The sine song, so called because of the continuous waveform that is produced when it is recorded and amplified, is made by the repeated vibration of a single wing. When amplified, the sine song sounds like a buzzing mosquito and, as the name suggests, there are no audibly distinct pulses.

Previous work has indicated that among many closely related species of Drosophila the IPIs of pulse songs differ in a species- specific way and therefore might play a central role in courtship and species recognition (Bennet-Clarke \& Ewing, 1968; Hoikkala \& Lumme, 1984; Ewing \& Miyan, 1986; Ritchie \& Gleason, 1995; Noor \& Aquadro, 1998). If species-specificity of IPIs is essential for intraspecific mate recognition, then IPI differences could also lead to sexual isolation among species. Using a playback experiment, von Schilcher (1976) showed that $D$. melanogaster females confined with a wingless conspecific male copulated more rapidly when subjected to a 34 millisecond IPI (characteristic of $D$. melanogaster) than when subjected to white noise. Furthermore, D. melanogaster females paired with wingless conspecific males and subjected to an artificial song with an IPI of $48 \mathrm{~ms}$ (mimicking the IPI of the sister species $D$. simulans) were slower to copulate than females subjected to an artificial song with the conspecific IPI of $34 \mathrm{~ms}$.

A ninth species, $D$. santomea, was recently discovered in the D. melanogaster subgroup (Lachaise et al., 2000). This species, endemic to the island of São Tomé, a $860 \mathrm{~km}^{2}$ volcanic island $255 \mathrm{~km}$ west of Gabon, is the sister species of the pan-African D. yakuba (Lachaise et al., 2000), and probably arose when the ancestor of $D$. yakuba colonized Sao Tome about 300,000 years ago (Llopart et al., 2002, 2005a). D. yakuba subsequently reinvaded the island, and now coexists with $D$. santomea. The two sibling species form a classic hybrid zone at middle elevations (the only hybrid zone known in the genus Drosophila), where there is a smooth transition between low-altitude populations consisting almost entirely of D. yakuba individuals and higheraltitude sites harboring only of $D$. santomea (Llopart et al., 2005a). Such hybrid zones have long been hailed as "windows of the evolutionary process", mainly because they allow one to dissect how different traits can produce barriers to gene flow and thereby contribute to the formation of new species. Previous studies (Llopart et al., 2005a, b) showed that in nature there is almost no gene flow across the D. yakuba/D. santomea hybrid zone, probably because of strong mate discrimination between these species as well as postzygotic barriers including hybrid male sterility (Coyne et al., 2002, 2005).

Here we characterize for the first time the male courtship song (pulse song) of $D$. santomea and compare it to that of its sister species D. yakuba. Unlike other species in the D. melanogaster subgroup, D. yakuba has no sine song (Cowling \& 


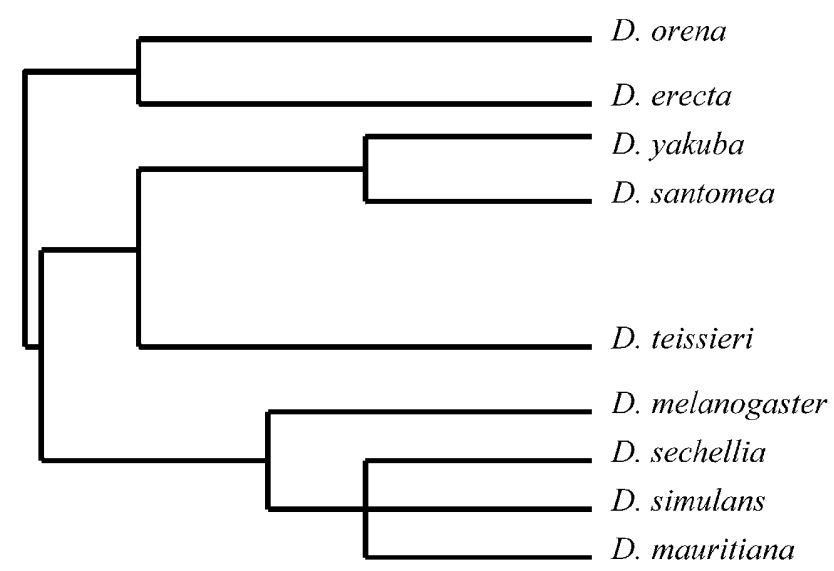

Fig. 1. Phylogeny of D. melanogaster subgroup, showing the sister-species relationship of D. yakuba and D. santomea. See Llopart et al. (2005a) for references on which this phylogeny is based.

Burnet, 1981), and has been reported to produce a pulse song of IPI approximately $120 \mathrm{~ms}$ (Demetriades et al., 1999). Since thud songs are usually produced when males are not oriented towards or in close proximity to the female, they do not have a clear role in courtship (Demetriades et al., 1999). Moreover a comparison of pulse and sine songs in D. melanogaster shows that only pulse song appears to affect mate choice and interspecific discrimination (Talyn \& Dowse 2004). Since the species discussed in this paper lack sine song, we recorded and measured only the parameters of the pulse song.

\section{MATERIAL AND METHODS}

\section{Drosophila stocks}

Flies were raised on standard cornmeal-yeast-agar medium at $24^{\circ} \mathrm{C}$ and kept in incubators on a 12-h light-dark cycle. Stocks of D. yakuba and D. santomea founded from single females captured in the wild were used as well as "synthetic" stocks (mixtures of isofemale lines) described below. In the latter stocks, several isofemale lines from a single population were combined to reconstitute the genetic variation of natural populations and eliminate the effects of any inbreeding that had occurred in the laboratory. (As the Results show, song parameters did not differ significantly between single or mixed isofemale lines.)

Two D. santomea stocks, STO.4 and 2005 SYN, were used in the analysis. The STO.4 stock was derived from a single inseminated female collected in the Obo Natural Reserve on São Tomé Island in 1998 within the zone of sympatry with $D$. yakuba (Lachaise et al., 2000). The 2005 SYN stock was derived from a combination of six isofemale lines collected by JAC in the zone of sympatry in January 2005.

We also used D. yakuba stocks Taï 18 and 2005 SYN in our analysis. The Taï 18 stock was collected in 1983 by D. Lachaise in the Taï rainforest on the border of Liberia and Ivory Coast. The D. yakuba stock 2005 SYN was made by combining six isofemale lines collected by JAC in the area of species overlap in January 2005.

Male hybrids for song analysis were produced by reciprocally crossing the 2005 SYN lines of D. santomea and D. yakuba.

\section{Mating behaviors of D. yakuba and D. santomea}

While not yet formally analyzed, the visible (non-auditory) courtship behaviors of $D$. santomea are, to the human eye, similar to those of D. yakuba. During their initial introduction into a mating chamber, males and females either remain motion-
TABLE 1. Differences in IPI between $D$. santomea and $D$. yakuba. The species mean IPI was calculated from the individual means within each species. The standard error provided is the standard error of the grand mean for each strain. Hybrids were generated by reciprocally crossing the 2005 SYN lines of D. santomea and D. yakuba.

\begin{tabular}{cccccc}
\hline Pure species & Line & $\mathrm{N}$ & Mean IPI (ms) & SE & Range \\
\hline \multirow{2}{*}{ D. santomea } & $2005 \mathrm{SYN}$ & 15 & 65.2 & 8.5 & 19.0 \\
& STO.4 & 10 & 68.0 & 6.1 & 22.2 \\
D. yakuba & $2005 \mathrm{SYN}$ & 15 & 105.9 & 12.7 & 38.6 \\
& Taï 18 & 10 & 113.3 & 11.1 & 37.7 \\
\hline Cross & Mother & $\mathrm{N}$ & Mean IPI (ms) & SE & Range \\
\hline $\mathrm{F}_{1}[\mathrm{~S} \mathrm{X} \mathrm{Y}]$ & santomea & 28 & 74.3 & 1.7 & 39.2 \\
$\mathrm{~F}_{1}[\mathrm{Y}$ X S] & yakuba & 29 & 80.4 & 1.8 & 39.5 \\
\hline
\end{tabular}

less or wander aimlessly. When the two flies come into contact, or close proximity, males approach the female while producing a thud song. If the female stops walking in the chamber, the male will approach her from behind and produce a pulse song. After a train of about 20 pulses, the male will attempt to copulate. If the first copulation attempt is unsuccessful, the male repeats this pattern until copulation is successful or the male breaks off courtship completely.

\section{Song analysis}

Flies used in the analysis were collected under $\mathrm{CO}_{2}$ anesthesia within $8 \mathrm{~h}$ of eclosion. Sexes were isolated and kept in vials of 15 flies. Only flies $4-6$ days old were used in the analysis. All songs were performed by aspirating a single male into an "Insectavox" song-recording device (Gorczyca \& Hall, 1987) containing a single conspecific female at a temperature of $23 \pm$ $2^{\circ} \mathrm{C}$. We amplified the recordings using a Radio Shack amplifier (Catalog no. 277-1008C) connected to a Macintosh Powerbook computer. The analog signals were digitized and basic song parameters analyzed by Canary sound analysis software (version 1.2.4, Cornell Bioacoustics).

For each male, we recorded every bout of pulse song produced, and used only one song for each male. Songs included in the analysis were over 10 pulses in length. Males who did not initiate courtship were discarded after 5 min while courting males were discarded after $10 \mathrm{~min}$. After each successful recording, the ambient temperature inside the Insectavox was measured using a thermocouple $\left( \pm 0.1^{\circ} \mathrm{C}\right)$.

To reduce the temperature effects on song parameters, we recorded songs only within a narrow temperature range $\left(4.0^{\circ}\right.$, from $21.2-25.2^{\circ} \mathrm{C}$ ) and also alternated recordings of the two pure species and the two reciprocal $F_{1}$ hybrids to reduce the effects systematic changes in temperature. The songs of the two types of $F_{1}$ males were assayed by placing them with a $D$. santomea 2005 SYN female. The males of both species readily court heterospecific females (Coyne et al., 2002).

\section{RESULTS}

Table 1 shows the differences in interpulse interval between the species and among the two strains tested within each species. The IPI was recorded for each song and a mean was found for each individual. The mean IPI for each strain was determined by averaging the individual means among males; the standard error given is the standard error among the means of different males tested in each strain. The difference in interpulse interval between these species is large and nonoverlapping. The mean IPIs for the two strains of D. santomea are $65.2 \mathrm{~ms}$ and $68.0 \mathrm{~ms}$, while those for the two strains of D. yakuba are 105.9 
TABLE 2. Analysis of variance for interpulse intervals, $D$. santomea vs. D. yakuba.

\begin{tabular}{lcccc}
\hline \multicolumn{1}{c}{ Source } & DF & Sum of Squares & F Ratio & Prob $>$ F \\
\hline Species & 1 & 20604.6 & 205.87 & $<0.0001$ \\
Strain (within species) & 2 & 219 & 1.0942 & 0.3434 \\
Error & 46 & 4603.9 & & \\
\hline
\end{tabular}

$\mathrm{ms}$ and $113.3 \mathrm{~ms}$. The mean IPIs of the species thus differ by about $43 \mathrm{~ms}$ - a difference far greater than seen between any other sister species in this subgroup. For instance, D. melanogaster and $D$. simulans have a difference of $14 \mathrm{~ms}$ between their IPI (Bennet-Clarke \& Ewing, 1968), while in the $D$. obscura group, the well-studied D. persimilis and D. pseudoobscura differ in IPI by only $18 \mathrm{~ms}$ (Noor \& Aquadro, 1998). (The IPIs of the melanogaster-group species are roughly $34 \mathrm{~ms}$ for $D$. melanogaster, 48 for D. simulans, $67 \mathrm{~ms}$ for D. sechellia, and $39 \mathrm{~ms}$ for D. mauritiana [Bennett-Clarke \& Ewing, 1968; Gleason \& Ritchie, 1994; Pugh \& Ritchie, 1996]).

Table 2 shows the nested analysis of variance for these data. The differences among strains within a species were not significant $\left(\mathrm{F}_{2,46}=0.34\right)$. The differences between the species, however, is highly significant $\left(\mathrm{F}_{1,2}=205.8, \mathrm{p}<0.0001\right)$. The IPI differences thus appear to be species-specific, as they are consistent among different strains of the same species collected in different years and locations.

Looking at the reciprocal $F_{1}$ males, one observes a slight (6.1 $\mathrm{ms})$ but significant effect of the X chromosome on mean IPI $(\mathrm{t}=$ $2.55,55$ d.f., $p=0.013)$. This difference is in the direction expected if the $\mathrm{X}$ chromosome carries genes contributing to the observed difference between the species, since those hybrids carrying the D. yakuba $\mathrm{X}$ chromosome have a larger mean IPI. However, the difference contributed by the $\mathrm{X}$ chromosome is a relatively small fraction of the total species difference in IPI (6.1/43 or about $14 \%)$. This $14 \%$ difference is roughly the same as the fraction of genetic material contained on the D. yakuba X chromosome - about $18 \%$. Thus the X chromosome does not have a disproportionately large effect on this species difference. In addition, there is some dominance of the D. santomea autosomes on the trait since both $F_{1}$ hybrids have an IPI closer to that of pure D. santomea males than to that of pure D. yakuba males. We did not find a significant correlation between temperature and mean IPI $(t=0.36$, 49 d.f., $p=0.72)$. Although such correlations have been seen in other species (Shorey, 1962), we used a much narrower temperature range.

\section{DISCUSSION}

Our study shows that D. santomea males, like their D. yakuba relatives (Cowling \& Burnet, 1981), lack a sine song and possess both a thud and a pulse song. These two species are the only ones lacking a sine song in the $D$. melanogaster subgroup, and thus its absence is probably a derived condition that evolved in the common ancestor of these two species (see Fig. 2).

During their courtship, Drosophila males communicate with females via visual, acoustic, olfactory, tactile, and gustatory sensory signals. Epicuticular hydrocarbons, fatty-acid derived hydrocarbons present on the Drosophila cuticle, have pheromonal activity in some species and are involved in mate recognition in D. mojavensis, D. arizonae, and D. navojoa (Etges \& Jackson, 2001), as well as D. simulans, D. melanogaster, D. sechellia, D. simulans (Coyne et al., 1994; Coyne, 1996; Coyne \& Charlesworth, 1997). However, recent studies by Llopart et al. (2002) indicate that it is unlikely that these hydrocarbons play a role in sexual isolation between $D$. yakuba and D. santomea, as their predominant epicuticular hydrocarbons are

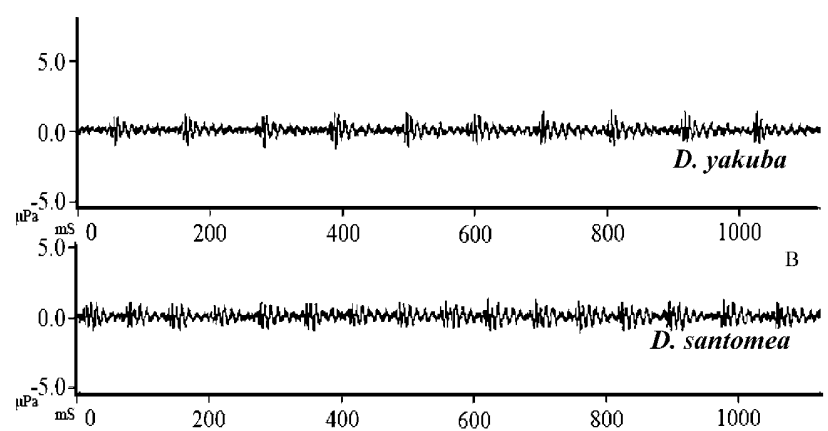

Fig. 2. Sample waveform recordings of D. yakuba and $D$. santomea courtship songs.

nearly identical. Furthermore, Llopart et al. (2002) also measured courtship behaviors under light and dark conditions and showed that the absence of light does not affect sexual isolation in these two species. This suggests that cues other than visual signals are required for the strong sexual isolation shown between $D$. yakuba and $D$, santomea.

Sexual isolation between these species derives largely from female $D$. santomea refusing the persistent courtship of $D$. yakuba males (Coyne et al., 2005). The difference in mean IPI between the species reported here - larger than that described in any pair of sibling species in this group - might play a role in this sexual selection. We are studying this possibility using artificial song.

Preliminary genetic analyses indicate that the $\mathrm{X}$ chromosome has an effect of $6.1 \mathrm{~ms}$ difference between reciprocal $\mathrm{F} 1 \mathrm{~s}$ on the IPI. This result is approximately that expected based on the relative size of the $\mathrm{X}$ chromosome compared to the rest of the genome. This is in contrast to some theories that predict that the $\mathrm{X}$ chromosome will carry most of the genes responsible for sexual dimorphisms (Rice, 1984). Future genetic work will focus on quantitative-trait-locus (QTL) analysis of IPI differences, with the goal of finding candidate loci.

Finally, it remains to be seen whether $D$. santomea and $D$. yakuba pulse songs possess Kyriacou-Hall cycles in the length of IPIs. There is doubt, however, whether such cycles could cause sexual isolation, as this would require females to detect and discriminate against a pattern that varies over a long period of time. Finally, although the thud song was not studied in this analysis, it is possible that it differs between the species in a way that may differentially affect intraspecific or interspecific communication.

\section{REFERENCES}

Bastock M. \& Manning A. 1955: The courtship of Drosophila melanogaster. Behaviour 8: 85-111.

BenNet-Clark H.C. \& Ewing A.W. 1967: Stimuli provided by courtship of male Drosophila melanogaster. Nature 215: 669-671.

Bennet-Clark H.C. \& Ewing A.W. 1968: The courtship songs of Drosophila. Behaviour 31: 288-301.

Cowling D.F. \& BuRNET B. 1981: Courtship songs and genetic control of their acoustic characteristics in sibling species of the Drosophila melanogaster sub-group. Behaviour 29: 924-935.

Coyne J.A. 1996: Genetics of differences in pheromonal hydrocarbons between Drosophila melanogaster and D. simulans. Genetics 143: 353-364.

Coyne J.A. \& Charlesworth B. 1997: Genetics of a pheromonal difference affecting sexual isolation between Drosophila mauritiana and D. sechellia. Genetics 145: 1015-1030. 
Coyne J.A. \& OrR H.A. 2004: Speciation. Sinauer Associates, Sunderland, Massachusetts, xiii +545 pp.

Coyne J.A., Mah K. \& Crittenden A. 1994: Genetics of a pheromonal difference contributing to reproductive isolation in Drosophila. Science 265: 1461-1464.

Coyne J.A., Kim S.Y., Chang A.S., Lachaise D. \& Elwyn S. 2002: Sexual isolation between two species with overlapping ranges: Drosophila santomea and D. yakuba. Evolution 56: $2424-2434$.

Coyne J.A., Elwyn S. \& Rolan-Alvarez E. 2005: Impact of experimental design on Drosophila sexual isolation studies: direct effects and comparison to field hybridization data. Evolution 59: 2588-2601.

Demetriades M.C., Thackeray J.R. \& Kyriacou C.P. 1999: Courtship song rhythms in Drosophila yakuba. Anim. Behav. 57: $379-386$

Etges W.J. \& JACKsON L.L. 2001: Premating isolation is determined by larval rearing substrates in cactophilic Drosophila mojavensis. VI. Epicuticular hydrocarbon variation in Drosophila mojavensis cluster species. J. Chem. Ecol. 27: 2125-2149.

Ewing A.W. \& Miyan J.A. 1986: Sexual selection, sexual isolation and the evolution of song in the Drosophila repleta group of species. Anim. Behav. 34: 421-429.

Gleason J.M. \& Ritchie M.G. 2004: Do quantitative trait loci (QTL) for a courtship song difference between Drosophila simulans and D. sechellia coincide with candidate genes and intraspecific QTL? Genetics 166: 1303-1311.

Gorcyca M. \& Hall J.C. 1987: The INSECTAVOX, an integrated device for recording and amplifying courtship song. Drosoph. Inf. Serv. 66: 157-160.

Hoikkala A. \& Lumme J. 1984: Genetic control of the difference in male courtship sound between D. virilis and D. lummei. Behav. Genet. 14: 827-845.

KyRIACOU C.P. \& HaLl J.C. 1982: The function of courtship song rhythms in Drosophila. Anim. Behav. 30: 794-801.

Lachaise D., Harry M., Solignac M., Lemeunier F., Bénassi V. \& CARIOU M.-L. 2000: Evolutionary novelties in islands: Dro- sophila santomea a new melanogaster sister species from São Tomé. Proc. R. Soc. London (B) 672: 1487-1495.

Llopart A., Elwyn S., Lachaise D. \& Coyne J.A. 2002: Genetics of a difference in pigmentation between Drosophila yakuba and D. santomea. Evolution 56: 2262-2277.

Llopart A., Lachaise D. \& Coyne J.A. 2005a: An anomalous hybrid zone in Drosophila. Evolution 59: 2602-2607.

Llopart A., Coyne J.A. \& Lachaise D. 2005b: Multilocus analysis of introgression between two sympatric sister species of Drosophila, D. yakuba and D. santomea. Genetics 171: 197-210.

Noor M.A.F. \& Aquadro C.F. 1998: Courtship songs of Drosophila pseudoobscura and D. persimilis: analysis of variation. Anim. Behav. 56: 115-125.

Pugh A.R.G. \& Ritchie M.G. 1996: Polygenic control of a mating signal in Drosophila. Heredity 77: 378-382.

RicE W.R. 1984: Sex chromosomes and the evolution of sexual dimorphism. Evolution 38: 735-742.

Ritchie M.G. \& Gleason J.M. 1995: Rapid evolution of courtship song pattern in Drosophila willistoni sibling species. $J$. Evol. Biol. 8: 463-479.

SHorey H.H. 1962: Nature of sound produced by Drosophila melanogaster during courtship. Science 137: 677.

SPIETH H.T. 1952: Mating behaviour within the genus Drosophila (Diptera). Bull. Am. Mus. Nat. Hist. 99: 401-474.

StURTEVANT A.H. 1915: Experiments on sex recognition and the problem of sexual selection in Drosophila. J. Anim. Behav. 5: 351-366.

TALYN B.C. \& Dowse H.B. 2004: The role of courtship song in sexual selection and species recognition by female Drosophila melanogaster. Anim. Behav. 68: 1165-1180.

TAuber E. \& Eberl D.F. 2003: Acoustic communication in Drosophila. Behav. Proces. 64: 197-210.

VON SCHILCHER F. 1976: The function of pulse song and sine song in the courtship of Drosophila melanogaster. Anim. Behav. 24: 622-625.

Received August 8, 2006; revised and accepted August 30, 2006 